\title{
Contribuciones del Laboratorio de Cultivo de Tejidos Vegetales de Zamorano a la Micropropagación y Propagación de Plantas Libres de Patógenos
}

\author{
María Alexandra Bravo y Dinie Espinal de Rueda ${ }^{2}$
}

\begin{abstract}
Resumen. Zamorano inauguró el Laboratorio de Cultivo de Tejidos Vegetales en 1985, durante 27 años se ha investigado sobre propagación in vitro de cultivos ornamentales, agronómicos y maderables. Entre los ornamentales más investigados está la orquídea Rhyncholaelia digbyana, flor nacional de Honduras, y varias especies nativas de la región. También se ha trabajado en violeta africana (Saintpaulia ionantha), zamias (Zamioculcas zamiifolia), helechos (Nephrolepis cordifolia) y anturios (Anthurium andreanum), cultivos apreciados en el mercado local de ornamentales y que son producto de las prácticas del aprender haciendo de nuestros estudiantes. Los últimos cuatro años se ha investigado y producido plántulas de especies económicamente importantes para la industria y la producción de energía como la caña de azúcar y el Arundo donax; para procesos de extracción de compuestos como la Stevia rebaudiana; para la exportación como malanga (Colocasia esculenta), y camote (Ipomea batatas). Se ha contribuido con los pequeños y medianos productores y exportadores de camote con aproximadamente 3000 plantas por año de camote libres de virus.
\end{abstract}

Palabras clave: Arundo donax, camote, caña de azúcar, cultivo in vitro, malanga, ornamentales, orquídeas, pastos, propagación masiva, stevia.

\section{Contribution of the Plant Tissue Culture Laboratory of Zamorano to the Micropropagation and the Production of Pathogen Free Plants}

\begin{abstract}
Zamorano inaugurated the Plant Tissue Culture Laboratory in 1985. For 27 years the Lab has done research on in vitro propagation of ornamentals, agronomic and woody crops. Among the ornamentals, orchids like the Honduran national flower (Rhyncholaelia digbyana), has been produced successfully, as well as other native orchids, African violets (Saintpaulia ionantha), ferns (Nephrolepis cordifolia), anturiums (Anthurium andreanum) and Zamioculcas zamiifolia. All of these crops are highly appreciated in the local market and are the product of the learning by doing practices with our students. For the last four years the lab's focus has been directed on some economically important crops for industry and energy production, such as sugar cane and Arundo donax; for the compound-extraction industry with Stevia rebaudiana, and on taro root (Colocasia esculenta) and virus free sweet potato (Ipomea batatas) for farmers and exporters.
\end{abstract}

Key words: Arundo donax, in vitro culture, massive propagation, ornamentals, orchids, stevia, sugar cane, sweet potato, taro.

\section{Introducción}

En 1984, con el objetivo de mejorar la enseñanza de las ciencias e investigación, Zamorano inició la construcción de cuatro laboratorios, BotánicaBiología, Química, Física y Cultivo de Tejidos Vegetales. El Laboratorio de Cultivo de Tejidos Vegetales sería utilizado básicamente para investigación y tendría capacidad para 10 estudiantes a nivel avanzado. La construcción del laboratorio fue terminada un año después y una vez que llegaron los equipos se puso en operación (Malo 1985). El laboratorio formó parte del Departamento de Agronomía y hasta 1998 estuvo a cargo del Doctor Juan José Alán, especialista en fitomejoramiento, genética y cultivo de tejidos (Malo 1984).

Durante los siguientes cinco años, en el laboratorio de cultivo de tejidos se desarrollaron actividades de investigación, enseñanza y producción. En 1989 se publicó la primera tesis producto de la investigación realizada en el laboratorio (Rodríguez Navarrete 1989). Este trabajo fue con Passiflora

\footnotetext{
${ }^{1}$ M.Sc. Encargada del Laboratorio de Cultivo de Tejidos Vegetales e Instructora, Escuela Agrícola Panamericana, Zamorano, Honduras. Correo electronico mbravo@zamorano.edu

${ }^{2}$ M.Sc. Profesora Asistente Fisiología Vegetal, Escuela Agrícola Panamericana, Zamorano, Honduras. Correo electrónico drueda@zamorano.edu
} 
quadrangularis, conocida como badea en los países andinos. En 1990 se reportó el éxito en la reproducción de varias especies ornamentales como violeta africana (Saintpaulia ionantha), gloxíneas (Sinningia speciosa), begonias y orquídeas. En el mismo año se adiciona un invernadero con lo que se incrementaron los trabajos de investigación (Escuela Agrícola Panamericana 1991). Las investigaciones realizadas por los estudiantes del Programa de Ingeniería Agronómica incluyeron temas relacionados al cultivo de tejidos en palma africana (Elaeis guineensis) (Rodríguez Ibañez 1992), bambú (Dendrocalamus asper) (Montalván Solorzano 1992), y arroz (Larco Vera 1992).

En 1994 le siguieron trabajos con especies ornamentales monocotiledóneas (Fajardo Oliva 1994) y la producción de híbridos de Phaseolus vulgaris y $P$. acutifolius en retrocruzas congruentes mediante el rescate de embriones (Pérez Camacho 1994). En 1997 y 1998 se estaban realizando más trabajos con zingiberáceas (Zelaya Zamorano 1997 y Peñaherrera Palacios 1998), y los helechos ornamentales Nephrolepis exaltata cv. Bostoniensis (helecho bostoniensis) y $N$. cordigera (helecho cola de quetzal) (Espinal y Alán 1997 y Castro Doomernik 1999).

Las investigaciones realizadas durante los 27 años de existencia del laboratorio han sido aplicadas en la propagación masiva de especies agronómicas de interés comercial y especialmente en la producción de plantas libres de patógenos. En el 2010 se inició la producción de plántulas de cultivos agronómicos para la empresa privada y asociaciones de exportadores que requieren de plántulas de camote y malanga libre de virus en Honduras y Nicaragua y se continúa con la producción de ornamentales como parte de las prácticas regulares de los estudiantes de módulo. También, a partir de este año se ofrece el servicio de adaptación y validación de protocolos de micropropagación a escala experimental.

El laboratorio cuenta con siete estaciones de flujo laminar y un área de incubación de $36 \mathrm{~m}^{2}$, está equipado con los aparatos esenciales para un laboratorio de enseñanza e investigación para el cultivo de tejidos vegetales y de micropropagación convencional. Además de las actividades de educación, se ofrece servicios de micropropagación a pequeña escala de plantas libres de patógenos.
También se atiende la demanda creciente de la industria local en el área de investigación, y adaptación y desarrollo de protocolos de micropropagación a nivel experimental.

\section{Propagación de la Orquídea Rhyncholaelia digbyana}

La investigación para la propagación de la Rhyncholaelia digbyana, flor nacional de Honduras, se inició en Zamorano en 1990 (Escuela Agrícola Panamericana 1991). Linares y Alán, en 1993, publican un artículo sobre germinación asimbiótica de embriones de orquídeas (Rosas 1993). El primer trabajo publicado de la orquídea $R$. digbyana corresponde a José Ledis Linares (Linares 1993), quién bajo la dirección del Dr. Juan José Alan, desarrolló un exhaustivo estudio para desarrollar un método adecuado de micropropagación que garantizase un abastecimiento de vitro-plántulas que puedan ser utilizadas para la hibridación y repoblación de hábitats naturales de la especie.

Dinie Espinal de Rueda estuvo a cargo del laboratorio de 1998 hasta el 2009 y continuó con las investigaciones en orquídeas, dándole especial importancia a la propagación de $R$. digbyana. Durante este periodo se llevaron a cabo varios estudios para evaluar la supervivencia en campo de las plántulas producidas in vitro (Mejía Bonilla 1999); determinar costos de producción (Lara Chávez 2001); evaluar tasas de multiplicación (Salgado Moncada 2002); y estudios para mejorar la sobrevivencia de plántulas que serían transferidas al invernadero donde serían aclimatadas (Córdova Yepez 2003). Estos estudios fueron la base para que en el 2004, con el apoyo de la Fundación Maya, se hiciera una introducción masiva en el Parque Arqueológico de Copán Ruinas de plantas aclimatadas. Durante estos años se han producido cientos de plantas que son destinadas a la repoblación en bosques y para el ornato de casas y jardines (Escuela Agrícola Panamericana 2006).

\section{Producción de Plantas Libres de Patógenos: Aráceas Comestible, Camote y Yuca}

En las aráceas comestibles, como la malanga (Colocasia esculenta), se evaluaron protocolos 
experimentales, haciendo los ajustes necesarios para lograr optimizar la propagación y la producción de plantas libres de patógenos a partir de meristemos apicales, logrando producir en un año unas 20,000 plántulas. Los resultados de estas evaluaciones sirvió para la apertura de un laboratorio de cultivo de tejidos en Nicaragua para la producción de plántulas libres de patógenos (Pitty y Cuevas García 2012).

La producción de camote para el Proyecto de Manejo Integrado de Plagas de Centroamérica (PROMIPAC) se inició en el 2010 con fondos de los Proyectos de Apoyo a la Investigación Colaborativa (CRSP por sus siglas en inglés). Esta producción se basó en los protocolos experimentales de la investigación de Gil Villacís (2005). También se contó con la colaboración de investigadores de Zamorano, la Fundación Hondureña para la Investigación Agrícola (FHIA) y de universidades de Norteamérica para las pruebas de detección de virus en los explantes establecidos. Durante estos tres años se han entregado unas 9,000 plántulas de camote libres de virus a pequeños y medianos productores hondureños que usarán estas plantas como fuente de material vegetal sano para propagación. También se han adaptado protocolos para la producción de plantas de yuca libres de virus (Buechsel Reyes 2012).

\section{Micropropagación de Caña de Azúcar y otras Especies para Generación de Energía}

El interés de obtener plantas libres de patógenos y asegurar la propagación de variedades élites seleccionadas es creciente en la industria azucarera de Honduras y Nicaragua. Los esfuerzos por servir a esta industria se iniciaron con investigaciones básicas en 1996 (Peña Paniagua 1997, Martínez Vega 2005, Araya Contreras 2006 y Gómez Pineda 2007). Debido al gran interés de la industria azucarera local y con su apoyo en el 2009, se capacitó al personal del laboratorio a través de un intercambio con la Universidad Nacional de El Salvador. Durante el 2010 y 2011 se produjeron plántulas de cinco variedades seleccionadas por la compañía azucarera, estas fueron trasplantadas al campo donde se evaluó su estabilidad genética, teniendo buenos resultados. Durante el 2012 se continuó con investigaciones para la micropropagación de otras dos variedades de caña
(Parreño Humanante 2012 y Zuñiga Pinto 2012).

También se ha desarrollado un protocolo para la micropropagación de Arundo donax (de León de León 2012). Esta especie será utilizada por la industria azucarera para la generación de energía en los periodos interzafra. Adicionalmente, se realizan esfuerzos para la micropropagación de Jatropha curcas, planta de cuyas semillas se extrae aceite para la elaboración de biodiesel. De esta especie existen numerosos protocolos publicados, hasta ahora ninguno con resultados repetibles para la formación de órganos. En el 2011, con la investigación de Almeida se lograron establecer cultivos callogénicos (Almeida Montenegro 2011). Durante el 2012 se llegó al desarrollo de brotes y se continuará con la investigación hasta llevar plantas a campo.

\section{Propagación Masiva de Stevia rebaudiana B.}

La stevia es una planta nativa del Paraguay de la que se extrae edulcorantes no calóricos, esta tiene actualmente una demanda creciente en la industria local para la extracción de steviosidos. La investigación para la micropropagación de Stevia rebaudiana a partir de segmentos nodales se inició en el 2001 a través de los trabajos realizados por Delvalle Báez (2001) y Ramia Avennatti (2002). Mediante estas investigaciones se logró elaborar el protocolo completo de reproducción in vitro para esta especie. Anualmente estamos propagando mil plántulas que serán usadas en la industria local.

\section{Otros Cultivos Ornamentales, Agronómicos y Maderables}

El laboratorio se ha destacado por el apoyo a productores y exportadores como es los trabajos con Zamioculcas zamiifolia (Hernández Sermeño 2005 y Díaz Aguirre 2007) y Sansevieria trifasciata (Ronquillo López 2005 y Vallejo Rendón 2007), rosa miniatura (Paz Castillo 2000), anturios (Ruiz Ruiz 2000), y helechos (Oliva Chávez 2003). Además, se ha apoyado a los productores de plátano (Reyes Padilla 2001 y Calla Zalles 2002); vainilla (Córdova Mollinedo 2007); moringa (Artiga Suárez 2012) y abeto (Pérez Gómez y Ramos Martín 2012). Igualmente, los trabajos in vitro con materiales de 
maderables altamente recalcitrantes, pero que fueron objeto de investigación: caoba (Manosalvas Scacco 2001), Tabebuia guayacan (Espinoza Dassum 2001), Tabebuia rosea (Pavón Banegas 2002) y Magnolia yoroconte (Llive Cóndor 2005).

\section{Literatura Citada}

Almeida Montenegro, A.D. 2011. Establecimiento in vitro de Jatropha curcas L. a partir de hojas cotiledonares y su respuesta a 6-Benciladenina y Ácido indol-3butírico. Proyecto especial de graduación del programa de Ingeniería Agronómica, Escuela Agrícola Panamericana, Zamorano, Honduras. $16 \mathrm{p}$.

Artiga Suárez, M.E. 2012. Efecto del BAP y 2,4-D en la inducción in vitro de tejido callogénicos a partir de láminas foliares, segmentos peciolares y vitroexplantes hipocotiledonares y radiculares de Moringa oleifera. Tesis Ing. Agr., Escuela Agrícola Panamericana, Zamorano, Honduras. $25 \mathrm{p}$.

Araya Contreras, J. 2006. Establecimiento in vitro de cuatro variedades de caña de azúcar a partir de explantes foliares y yemas axilares. Tesis Ing. Agr., Escuela Agrícola Panamericana, Zamorano, Honduras. 22 p.

Bueschel Reyes, C.D. 2012. Establecimiento in vitro de yuca -variedad valencia- mediante domos meristemáticos y evaluación de tres medios de cultivo para la producción de brotes. Proyecto especial de graduación del programa de Ingeniería Agronómica, Escuela Agrícola Panamericana, Zamorano. Honduras. 18 p.

Calla Zalles, B. 2002. Efectos del uso de Mycoral ${ }^{\circledR}$ durante la aclimatación y endurecimiento de plátano (Musa spp.) Cuerno y FHIA-20 producidos a partir de ápices meristemáticos. Tesis Ing. Agr., Escuela Agrícola Panamericana, Zamorano, Honduras. 33 p.

Castro Doomernik, A. 1999. Aclimatación de dos especies de helecho propagadas in vitro: Nephrolepis exaltata cv. Bostoniensis (helecho bostoniensis) y Nephrolepis cordigera (helecho cola de quetzal). Tesis Ing. Agr., Escuela Agrícola Panamericana, Zamorano, Honduras. $35 \mathrm{p}$.

Córdova Mollinedo, D. 2007. Reproducción in vitro de vainilla (Vanilla planifolia A.) a partir de segmentos nodales y yemas axilares. Tesis Ing. Agr., Escuela Agrícola Panamericana, El Zamorano, Honduras. 15 p.

Córdova Yepez, J. 2003. Evaluación de seis substratos y Mycoral ${ }^{\circledR}$ durante la aclimatación de vitroplántulas de la orquídea Rhyncholaelia digbyana. Tesis Ing. Agr., Escuela Agrícola Panamericana, Zamorano, Honduras. $46 \mathrm{p}$. de León de León, C.A. E. 2012. Micropropagación de Arundo donax a partir de yermas axilares y meristemos apicales. Proyecto especial de graduación del programa de Ingeniería Agronómica, Escuela Agrícola Panamericana, Zamorano. Honduras. 27 p.

Delvalle Báez, W.E. 2001. Propagación in vitro de Stevia rebaudiana $\mathrm{B}$. a partir de segmentos nodales. Proyecto Especial del Programa de Ing. Agr., Zamorano, Honduras. $44 \mathrm{p}$.

Díaz Aguirre, F. 2007. Respuesta regenerativa in vitro de Zamioculcas zamiifolia (Fam. Araceae) a partir de explantes foliares. Tesis Ing. Agr., Escuela Agrícola Panamericana, Zamorano, Honduras. 30 p.

Escuela Agrícola Panamericana. 1991. Informe Anual 19901991. Escuela Agrícola Panamericana, Zamorano, Honduras. 37 p.

Escuela Agrícola Panamericana. 2006. Informe anual. Escuela Agrícola Panamericana, Zamorano, Honduras. $29 \mathrm{p}$.

Espinal D. y J.J Alán. 1997. Propagación in vitro del helecho cola de quetzal. In J.C. Rosas (ed.) Informe anual de investigación Departamento de Agronomía1996. Escuela Agrícola Panamericana. Zamorano. Honduras. Volumen 9: 36-38.

Espinoza Dassum, C. 2001. Evaluación del uso de ápices meristemáticos y de explantes cotiledonares e hipocotiledonares en el establecimiento in vitro de Tabebuia guayacán (Cortés). Tesis Ing. Agr., Escuela Agrícola Panamericana, Zamorano, Honduras. 38 p.

Fajardo Oliva, E. 1994. Establecimiento in vitro de tres especies de ornamentales monocotiledóneas. Tesis Ing. Agr., Escuela Agrícola Panamericana, Zamorano, Honduras. $56 \mathrm{p}$.

Gil Villacis, D.M. 2005. Micropropagación de vitroplantas de camote (Ipomoea batatas) variedad Bush bock establecidas a partir de meristemas. Proyecto Especial del Programa Ing. Agr. en Ciencia y Producción Agropecuaria, Zamorano, Honduras. 23p.

Gómez Pineda, J. 2007. Efecto de tratamientos antioxidantes en el establecimiento in vitro de dos genotipos de caña de azúcar del Ingenio La Grecia, Honduras. Tesis Ing. Agr., Escuela Agrícola Panamericana, Zamorano, Honduras. $14 \mathrm{p}$.

Hernández Sermeño, V. 2005. Organogénesis indirecta in vitro de Zamioculcas zamiifolia (Fam. Araceae). Tesis Ing. Agr., Escuela Agrícola Panamericana, Zamorano, Honduras. $40 \mathrm{p}$.

Lara Chávez, A. 2001. Análisis de costos de producción in vitro y de mercado de orquídeas en Zamorano. Tesis Ing. Agr., Escuela Agrícola Panamericana, Zamorano, Honduras. $60 \mathrm{p}$. 
Larco Vera, A. 1992. Producción de haploides de arroz por medio del cultivo de anteras. Tesis Ing. Agr., Escuela Agrícola Panamericana, Zamorano, Honduras. 63 p.

Linares, J.L. y J.J. Alán. 1993. Germinación Asimbiótica de Embriones de Algunas Especies e Híbridos de Orquídeas. In J.C. Rosas (ed.) Informe anual de investigación Departamento de Agronomía-1992. Escuela Agrícola Panamericana. Zamorano. Honduras. Volumen 5: 123-127.

Linares, J.L. 1993. Propagación in vitro de la orquídea Rhyncholaelia digbyana (Lindl.) Schltr. Proyecto especial de Ing. Agr., Zamorano, Honduras. 88 p.

Llive Cóndor, F. 2005. Callogénesis in vitro de El Redondo (Magnolia yoroconte Dandy) a partir de la siembra apolar de explantes foliares. Tesis Ing. Agr., Escuela Agrícola Panamericana, Zamorano, Honduras. 24 p.

Malo, S. 1984. Informe del Director. In Informe Anual. Escuela Agrícola Panamericana, Zamorano, Honduras. $24 \mathrm{p}$.

Malo, S. 1985. Informe del Director. In Informe Anual. Escuela Agrícola Panamericana, Zamorano, Honduras.22 p.

Manosalvas Scacco, E.D. 2001. Estudio del riesgo técnicofinanciero para la producción in vitro y plantación de clones de Swietenia macrophylla (caoba) en Honduras. Tesis Ing. Agr., Escuela Agrícola Panamericana, Zamorano, Honduras. $61 \mathrm{p}$.

Martínez Vega, A. 2005. Elaboración de un procedimiento de desinfección y establecimiento in vitro de caña de azúcar, variedad CP 72-2086 a partir de yemas axilares. Tesis Ing. Agr., Escuela Agrícola Panamericana, Zamorano, Honduras. 39 p.

Mejía Bonilla, I. A. 1999. Evaluación de la supervivencia en el campo, de la flor nacional de Honduras, Rhyncholaelia digbyana, Lindl, Schltr, variedad fimbripetala Ames, de origen in vitro, en Zamorano, valle del Yeguare, Honduras. Tesis Ing. Agr., Escuela Agrícola Panamericana, Zamorano, Honduras. 23 p.

Montalván Solórzano, P. 1992. Propagación vegetativa in vitro del bambú. Tesis Ing. Agr., Escuela Agrícola Panamericana, Zamorano, Honduras. 65 p.

Oliva Chávez, A. 2003. Producción in vitro de helecho cola de quetzal (Nephrolepis cordifolia) comparando y evaluando los costos de dos sistemas de multiplicación y dos concentraciones de kinetina. Tesis Ing. Agr., Escuela Agrícola Panamericana, Zamorano, Honduras. $38 \mathrm{p}$.
Parreño Humanante, J.G. 2012. Establecimiento in vitro de caña de azúcar (Saccharum officinarum) -variedad CP 72-2086-. Tesis Ing. Agr., Escuela Agrícola Panamericana, Zamorano, Honduras. 23 p.

Pavón Banegas, C. 2002. Evaluación del uso de ápices meristemáticos y explantes cotiledonares e hipocotiledonares en el establecimiento in vitro de Tabebuia rosea (Bertol) DC (macuelizo). Tesis Ing. Agr., Escuela Agrícola Panamericana, Zamorano, Honduras. 53 p.

Paz Castillo, M.P. 2000. Ensayo agronómico para el establecimiento de Rosa multiflora Thund. in vitro. Tesis Ing. Agr., Escuela Agrícola Panamericana, Zamorano, Honduras. $61 \mathrm{p}$.

Peñaherrera Palacios, J. 1998. Propagación in vitro de jengibre. Proyecto especial del programa de Ing. Agr., Escuela Agrícola Panamericana, Zamorano, Honduras. $29 \mathrm{p}$.

Peña Paniagua, M. 1997. Propagación in vitro de la caña de azúcar. Tesis Ing. Agr., Escuela Agrícola Panamericana, Zamorano, Honduras. 39 p.

Pérez Camacho, L. 1994. Producción de híbridos e incremento en la compatibilidad de Phaseolus vulgaris $\times$ Phaseolus scutifolius en retrocruzas congruentes mediante rescate de embriones. Tesis Ing. Agr., Escuela Agrícola Panamericana, Zamorano, Honduras. $97 \mathrm{p}$.

Pérez Gómez J.M. y S.D. Ramos Martín. 2012. Establecimiento in vitro de Abies guatemalensis (Pinabete) a partir de embriones cigóticos. Tesis Ing. Agr., Escuela Agrícola Panamericana, Zamorano, Honduras. 22 p.

Pitty, A. y R. Cuevas García. 2012. Informe Anual 2011. Escuela Agrícola Panamericana, Zamorano, Honduras. $40 \mathrm{p}$.

Ramia Avenatti, N.C. 2002. Estudio económico para la producción y comercialización de Stevia rebaudiana. Tesis Ing. Agr., Escuela Agrícola Panamericana, Zamorano, Honduras. 47 p.

Reyes Padilla, B.A. 2001. Uso de L-cisteína y ácido ascórbico para el control de la oxidación durante el establecimiento y la multiplicación in vitro de ápices meristemáticos de tres cultivares de plátano (Musa spp.) incubados bajo condiciones de luz y oscuridad. Tesis Ing. Agr., Escuela Agrícola Panamericana, Zamorano, Honduras. 52 p. 
Rodríguez Ibañez, D. 1992. Propagación in vitro de palma africana. Tesis Ing. Agr., Escuela Agrícola Panamericana, Zamorano, Honduras. 58 p.

Rodríguez Navarrete, S. 1989. Cultivo in vitro de Passiflora quadrangularis L. Tesis Ing. Agr., Escuela Agrícola Panamericana, Zamorano, Honduras. 39 p.

Ronquillo López, M.G. 2005. Inducción de callogénesis in vitro a partir de láminas foliares de Sansevieria trifasciata. Tesis Ing. Agr., Escuela Agrícola Panamericana, Zamorano, Honduras. 19 p.

Rosas, J.C. 1993. Informe anual de investigación Departamento de Agronomía-1992. Escuela Agrícola Panamericana, Zamorano, Honduras. 127 p.

Ruiz Ruiz, B. 2000. Efecto del BAP y 2,4-D en la inducción de organogénesis indirecta in vitro de Anthurium andraeanum L. Tesis Ing. Agr., Escuela Agrícola Panamericana, Zamorano, Honduras. $36 \mathrm{p}$
Salgado Moncada, J.D. 2002. Análisis del costo de producción y evaluación de la tasa de multiplicación in vitro de Rhyncholaelia digbyana en Zamorano. Tesis Ing. Agr., Escuela Agrícola Panamericana, Zamorano, Honduras. 57 p.

Vallejo Rendón, C.F. 2007. Multiplicación in vitro de Sansevieria trifasciata utilizando vitroláminas foliares y tejido callogénico. Tesis Ing. Agr., Escuela Agrícola Panamericana, Zamorano, Honduras. 32 p.

Zelaya Zamora, L.P. 1997. Propagación in vitro de zingiberaceas. Tesis Ing. Agr., Escuela Agrícola Panamericana, Zamorano, Honduras. 39 p.

Zuñiga Pinto, A.I. 2012. Establecimiento in vitro caña de azúcar (Saccharum officinarum) -variedad CP 731547. Tesis Ing. Agr., Escuela Agrícola Panamericana, Zamorano, Honduras. 25 p.

Recibido para publicación el 4 de febrero del 2013. Aceptado para publicación el 17 de junio del 2013. 